\title{
EL PRINCIPIO DE EQUIDAD \\ EN EL NOVUS ORDO POLITICO \\ Y LEGISLATIVO DEL SIGLO IV
}

\author{
Alberto PEREZ VIVO \\ Profesor Adjunto de Derecho Romano
}

Resumen de la tesis doctoral que bajo el mismo título, fue leída en la Facultad de Derecho de la Universidad de Alicante, el 14 de julio de 1982. La misma ha sido dirigida por el Prof. Dr. D. Jesús Daza Martínez y juzgada por el tribunal compuesto por los profesores: Dr. D. José Luis Iglesias Buhigues, Catedrático de Derecho Internacional de la Universidad de Alicante; Dr. D. Alfredo Calonge Matallanes, Catedrático de Derecho Romano de la Universidad de Salamanca; Dr. D. Antonio Ortega y Carrillo de Albornoz, Catedrático de Derecho Romano de la Universidad de Málaga; Dr. D. Jesús Daza Martínez, Catedrático de Derecho Romano de la Universidad de Alicante y Dr. D. Pelayo de la Rosa Díaz, Profesor Agregado de Derecho Romano de la Universidad de Salamanca.

La tesis obtuvo la calificación de sobresaliente cum laude. 

La expresión aequitatis ratio es frecuente en las constituciones de Constantino. En alguna de ellas, como la célebre constitución del año 314, parece alcanzar incluso la categoría de norma general cuando el Emperador establece:

Placuit, in omnibus rebus, praecipuam esse iustitiae aequitatisque quam stricti iuris ratio.

El fin de la investigación consiste fundamentalmente en determinar las nuevas bases teóricas de la aequitas y su incidencia en la práctica jurídica, a partir de los primeros años del siglo IV, cuando se consolida el Dominado y se impone la concepción absolutista del poder imperial, al mismo tiempo que la jurisprudencia pierde su urôle» oficial, y las constituciones del emperador pasan a ser la fuente esencial del derecho.

Esto explica la singular relevancia que tiene en el desarrollo del estudio, el Codex Theodosianus, en el que contienen "Omnes edictales generalesque constitutiones" desde Constantino hasta Teodosio II.

La aequitas de este período no se identifica ya con la equidad clásica romana, cuyo significado fundamental era la idea de correspondencia entre el ius y el hecho o realidad concreta, dando lugar a aquel carácter retributivo, matemático y rígido, pero objetivo que constituye un elemento esencial de la mentalidad jurídica romana.

Ahora, son patentes las influencias de la filosofía griega, del mundo helenístico, de los derechos horientales y, por supuesto, del cristianismo, que fue favorecido abiertamente por la legislación imperial y tuvo, a su vez, una participación activa en los contenidos y en la orientación de ésta.

Por tratarse de una realidad compleja, en la que se entrecruzan, por una parte, ideas filosoficas, tradiciones juridicas y creencias religiosas $y$, por otra, situaciones concretas relacionadas con el derecho privado, el derecho público, las costumbres de los pueblos y la evolución misma de los principios que rigen la administración de la justicia, el método de trabajo debía ser necesariamente interdisciplinar. Por supuesto, no debe entenderse por tal la mera acumulación de conocimientos o datos tomados de distintas disciplinas, sino la búsqueda de sus conexiones profundas, intentando su integración y articulación en una síntesis coherente, y haciendo posible una auténtica y rigurosa comunicación de saberes. 
De acuerdo con estos criterios el plan de trabajo y el orden de exposición se han ajustado al esquema lógico siguiente:

En primer lugar, era conveniente tener presentes las dos posiciones más caracterizadas que han mantenido los romanistas, a proposito de la interpretación de las transformaciones evidentes que experimenta la aequitas romana en la época postclásica. La diferencia fundamental que las separa no es otra que el modo de entender y explicar la evolución misma del derecho romano.

En unos casos, se habla de un proceso lineal en la formación de este derecho, desde el siglo II a.C. hasta Justiniano, sin que sea preciso recurrir, para explicarlo, a factores externos a su propia dinámica. La prevalencia que adquirió la aequitas sobre el ius fue debida al desarrollo orgánico del derecho romano, siendo los factores más importantes de esa evolución, la ampliación de la cognitio extra ordinem, la desaparición del praetor y de las formulae, ya en la primera mitad del siglo IV. La equidad se convierte, finalmente, en la sustancia misma de todo el ordenamiento jurídico y pasa a ser el ius por excelencia o ius aequum.

El otro punto de vista defiende que en la época clásica la aequitas no se contraponía al ius, del cual era, en realidad, la norma interna y, a la vez, la meta que el derecho positivo aspiraba a conseguir. No había, pues, contraposición ente el ius aequum y el ius strictum, sino únicamente entre el derecho presente y el derecho ya no existente o derecho arcaico. Al perder su fuerza la tradición romana, por influjo de las doctrinas griegas, los derechos provinciales y el cristianismo, la equidad deja de regular el derecho y de estar a su servicio, para terminar finalmente prevaleciendo sobre él.

Esto supuesto, el capítulo primero estudia los rasgos fundamentales de la nueva situación histórica: Entre otros, y por su incidencia en la legislación, se destacan el intervencionismo estatal, la reforma administrativa, la presión fiscal, las tensiones entre los potentiores y los humiliores, la conflictividad política y las amenazas exteriores sobre el limes del imperio. Como es lógico, se da un relieve especial al hecho de la progresiva integración del cristianismo en la sociedad y en el Estado romano, asi como 
al movimiento de reacción protagonizado, entre otros, por Juliano, en nombre de la priscarum legum reverentia.

En este contexto, era también obligada la referencia al problema de la unidad jurídica del Imperio, que parecía quedar cuestionada desde el momento mismo de su división en las dos partes conocidas como Oriental y Occidental. Tampoco puede pasarse por alto que es entonces cuando la noción clásica de Res Publica, que era una noción concreta y no institucionalizada, es sustituida por una concepción abstracta del ente público; paralelamente, la antigua utilitas communis o utilitas omnium se transforma en utilitas publica, entendida como interés del Estado, el cual subordina a sus conveniencias propias las utilitates civium. Era lógico que, por ese camino, se introdujeran modificaciones en aspectos fundamentales del ordenamiento jurídico, y por tanto, en la concepción de la equidad y de su función en la vida social.

La aequitatis ratio adquiere un relieve singular en la legislación, al iniciarse el novus ordo de Constantino, que contenía ya germinalmente la casi totalidad de los cambios institucionales, que alcanzarían posteriormente su desarrollo pleno con Justiniano.

El capítulo segundo, pues, se centra en el estudio de las constituciones de Constantino, en las que se invoca estrictamente la aequitatis ratio o principio de equidad, con el fin de determinar el significado preciso que debe darse a este concepto, su relación con la noción clásica de equidad y las posibles innovaciones que, de un modo $u$ otro, haya podido introducir el emperador.

El punto de partida, como es evidente, debía ser la importante constitución del año 314, que contrapone al ius strictum la iustitiae aequitatisque ratio, que debe prevalecer sobre él. Teniendo en cuenta las más recientes aportaciones de la romanística, se relaciona esta constitución con otros textos legislativos del mismo año, intentando precisar si su interpretación confirma la idea de que se trata de una norma que tiene alcance original, o bien deberá entenderse que los compiladores de Justiniano, aislando determinadas afirmaciones de su contexto, fueron los responsables de un cambio profundo de su significado. 
En cualquier caso, la complejidad del problema se hace más patente al considerar que la legislación de Constantino, en su terminología y en sus contenidos tiene influencias ciertas y directas de pensadores cristianos que estuvieron muy estrechamente vinculados con el emperador, en especial el filósofo y jurista Lactancio. Sus concepciones de la iustitia y de la aequitas, están a la base de numerosas disposiciones legislativas y constituyen su fundamento teórịco e ideológico.

Hay que destacar, a este propósito, el esfuerzo de Lactancio por penetrar en el pensamiento jurídico clásico, al mismo tiempo que busca introducir elementos nuevos tomados de la tradición cristiana.

La igualdad esencial de todos los hombres, más radical que cualquier otro posible alegato en favor de una societas aequa es la idea básica que domina en su síntesis jurídico-política, completando así el alcance que daba Cicerón a la aequabilitas, que era la consecuencia más inmediata que se deducia de su peculiar forma de entender las relaciones entre el ius, aequitas y iustitia.

Los indicios de la influencia de Lactancio sobre los textos legislativos de Constantino han sido analizados ampliamente, entre otros, en la reciente obra de Amarelli "Vetustas-Innovatio", que ofrece una serie de datos pormenorizados y de paralelismos textuales muy esclarecedores, aunque tal vez no profundiza suficientemente en los planteamientos teóricos que están a la base de la nueva concepción doctrinal que se estaba elaborando.

El capítulo tercero completa los análisis anteriores relacionando la aequitatis ratio con una institución importante, sin precedentes en la época clásica, y que surge y se desarrolla durante el siglo IV: La Episcopalis Audientia.

El reconocimiento oficial de esta institución, que durante los siglos anteriores sólo tenía una finalidad religiosa, significo un avance notable en la penetración de los principios cristianos en la sociedad, al otorgarse al episcopado una función preeminente en la administración y control de la justicia. 
Desde un punto de vista político las decisiones de Constantino dejan abierto el problema de determinar los motivos reales que pudieron inspirarla; en el campo de la historia del derecho, el interés se ha centrado en el análisis de la naturaleza jurídica de la ínstitución, discutiéndose todavía hoy si se trataba de un mero arbitraje o puede hablarse de una verdadera iurisdictio.

En nuestro trabajo, se resumen los contenidos básicos de las disposiciones imperiales sobre la Episcopalis Audientia, para centrar posteriormente la atención en un aspecto particular de ésta: su posible influencia en la evolución de la aequitatis ratio y en la efectividad real de la misma en la vida social del Imperio, teniendo en cuenta que los episcopi no aplicaban solamente las normas de derecho romano, sino también otras tomadas de las tradiciones jurídicas de diferentes pueblos (egipcio, griego, hebreo, entre otros), y que hacían esto, manteniéndose dentro del marco de exigencias que se derivaban de aquella lex christianorum a la que apela explícitamente Constantino en el momento de reconocer oficialmente esta institución.

En este sentido, es importante la observación que hace Biondi a propósito de la episcopalis audientia: a saber, que su función peculiar consistía no sólo en la sustitución de un juez laico por un juez eclesiástico, sino, sobre todo, en la sustancia misma del derecho que en ella se invocaba y en los criterios que presidían la aplicación de las leyes.

En la última parte de este capítulo se intenta precisar las diferencias que existían entre la epiekeia cristiana y aquella otra que procedía de la tradición filosofica griega, en especial del sistema aristotélico; o entre la lex christianorum y la aequitas que era propia de la tradición romana. No es indiferente, por ejemplo que el propio Concilio de Nicea exhortara a los episcopi a juzgar "secundum legis et aequitatis praecepta"; o que la ley cunctos populos del año 380, exigiera que in negotiis audiendis, fidem ac sinceritatem geminam praebiturum, et sacerdotis et iudicis".

El mismo término audientia, que los historiadores del derecho consideran excesivamente vago e impreciso, tiene, en la literatura cristiana y en los textos legislativos de los emperadores de este período, un contenido nuevo muy rico que no encuentra precedentes en los textos jurídicos clási- 
cos y que revela su relación con el sistema de ideas que procede de la tradición bíblico-cristiana.

El capítulo IV estudia la aequitatis ratio o principio de equidad teniendo en cuenta su significación en orden a la configuración de una societas aequa.

En esta prespectiva, las doctrinas estoicas-desde las primeras formulaciones griegas hasta su desarrollo posterior en el período romano-, adquieren un relieve singular. Su influencia en el pensamiento jurídico y en el mismo cristianismo se hace patente al estudiar o analizar conceptos como ius naturale, naturalis ratio y aequitas naturalis que iban a tener una gran trascendencia en el desarrollo de la filosofía del derecho y del Estado en el mundo occidental.

En los estoicos, la concepción moral es la clave última del sistema. Precisamente en la llamada "época media del estoicismo", caracterizada por la tendencia sincretista, que Cicerón llevará hasta el límite, es cuando se produce una confusión entre el dikáion phisikọ́n de Aristóteles, que él situa en el mundo del derecho, y el nomos phisikós de la tradición estoica, esto es, entre el orden moral y el orden jurídico.

Partiendo de estas concepciones técnicas, y de su influencia en el Derecho romano y en el cristianismo, en este capítulo se explica la relación que tuvo el principio de equidad con el problema de la esclavitud. Aparte de señalar las aportaciones positivas de las formulaciones estoicas y de los principios cristianos, así como sus limitaciones e insuficiencias, se analiza el alcance real que tuvieron algunas disposiciones legales, en especial la institución de la manumissio in ecclesia, que constituye una pieza importante dentro de la política legislativa imperial del primer tercio del siglo IV.

Por último, la aequitatis ratio es estudiada en este capítulo en su conexión con el concepto de humanitas. Pese a la pluralidad y riqueza de este término, en el ámbito del derecho se identificó posteriormente humanitas con aequitas, viniendo a ser expresión de benignitas, clementia, pietas o charitas. Por este camino, como han observado los romanistas, la equidad se convirtió, en muchos casos, en el terreno práctico, en una justi- 
ficación del laxismo en el derecho y vino a ser una realidad incierta y evanescente, sin contornos definidos, favoreciendo, por tanto, el proceso de decadencia del ius.

Sin embargo es posible hacer un planteamiento diferente e interpretar la humanitas desde una perspectiva nueva. No se trata solo de intentar una mitigación del rigor del derecho positivo, o de introducir una consideración subjetiva del mismo, o, en fin, de resaltar la necesidad de atender a las situaciones concretas en la praxis jurídica. Lo importante, como intentamos poner de manifiesto en la parte final de este capítulo, es que, al invocar la humanitas, se está afirmando una nueva idea de hombre, una concepción diferente de las relaciones del indivíduo con la sociedad, basada en la primacía de la persona y del mundo de valores que le es propio. En esta dirección, la conciencia antropológica del hombre como ser personal, que constituye tal vez la aportación más importante del cristianismo a nuestra cultura, debe ser considerada, como puso de relieve Hegel, un hito en la configuración de la civilización occidental. Dentro de ello el Derecho- y la equidad que lo interpreta y lo aplica-es un factor más, al servicio de ese proceso que llamamos humanización.

Las conclusiones más importantes a que nos ha conducido la investigación pueden resumirse brevemente en las proposiciones siguientes:

$1 .^{\circ}$-Durante el siglo IV, las bases jurídicas continúan siendo, en ambas partes del Imperio, las que habían existido durante el período clásico. Son también como los factores más importantes que incidieron en la evolución del derecho y algunos hechos específicos de esta época, tales como la desaparición del procedimiento formular, la atenuación del formalismo y la fusión de las instituciones civiles y pretorias.

2. ${ }^{\circ}$ - La transformación de los conceptos de iustitia y aequitas durante el siglo IV, está condicionada profundamente por el hecho de que desde Diocleciano a Teodosio I, la legislación es la expresión evidente de un progresivo desarrollo hacia la organización unitaria del Estado, de su poder normativo y de su supremacía frente a los cives. Esto explica, entre otras cosas, por qué la utilitas publica sustituye la antigua utilitas communis o utilitas omnium, y se opone a ella. 
3. ${ }^{\circ}$-En la legislación de Constantino, el principio de equidad o aequitatis ratio, no es entendido igualmente siempre. En algunas constituciones, es evidente que la aequitas mantiene los significados propios de esta noción en la época clásica, tales como, «juicio de valor sobre un caso particular", o «igualdad de trato en casos iguales», o "excepción a una norma por razones particulares".

En otras ocasiones, la aequitas es considerada como principio inspirador del derecho, conforme a la concepción griega sistematizada ya por Aristóteles. Cuando esto ocurre, la equidad cumple diversas funciones: regula los casos particulares, que no están contemplados en la ley, corrigiendo a ésta si es necesario; ofrece un criterio hermenéutico para interpretarla de acuerdo con la voluntad del legislador, y, finalmente, aparece como principio creador de derecho, allí donde existe una laguna de ley escrita, a fin de hacer efectivas las exigencias de la justicia.

Finalmente, el influjo del pensamiento cristiano, sobre todo de las doctrinas de Lactancio, introduce elementos nuevos que hacen que la aequitas sea, en muchos casos, la expresión de los imperativos que se derivan de la lex christianorum.

4. ${ }^{\circ}$-El reconocimiento de la Episcopalis Audientia es la demostración más evidente de esta última forma de entender y aplicar la aequitatis ratio. En esta institución el recurso al Derecho romano-o a los derechos de otros pueblos - se hacía siempre teniendo presente la epiekeia bíblica, como ponen de relieve las afirmaciones explícitas de los episcopi, que eran los responsables inmediatos de su apliçación práctica.

$5 .^{\circ}$-A partir del siglo IV, el principio de equidad se constituyó en el factor más importante de humanización de las relaciones sociales.

La confluencia de las doctrinas estoicas, especialmente las romanas, con el cristianiismo, tuvo, pese a sus limitaciones consecuencias importantes en la evolución del derecho, y en la toma de conciencia de las condiciones reales que deben darse, para qúe exista una auténtica societas aequa.

El hecho de que la legislación de esta época tendiera a mejorar, por ejemplo, las condiciones de vida de un esclavo o a facilitar su liberación, acentuando el favor libertatis o creando instituciones como la manumissio 
in ecclesia no es lo fundamental. Tampoco lo es la apelación a la aequitas como medio para mitigar el rigor del ius o como exprésión de benignitas o charitas. El dato nuevo más trascendente consiste en que la aequitas se identifica entonces con la humanitas y ésta, a su vez, viene a ser la formulación ideal de una nueva concepción antropológica, a saber, aquella que afirma la convicción personal del hombre. A partir de ahí, será posible hablar de igualdad esencial entre los seres humanos, de primacla de la persona sobre las instituciones, de derechos fundamentales inviolables. No sin razón, Justiniano podrá referirse a este hecho singular hablando de una nova ratio humanitatis. 